\title{
Tribute to reviewers
}

WJPS is dependent on the "unknown" or known reviewers for their generous expenditure of the effort, valuable time, and expertise. We appreciate their support to this journal and it is their assistance that ensured the overall quality of the journal.

Aktaş, Selma
Al-Dairy, Alwaleed
Anand, Sachit
Angelico, Roberta
Arnbjornsson, Einar
Aronson, Daniel C
Asti, Lindsey
Avanzini, Stefano
Baird, Robert
Barrett, Cindy S
Bass, Lee M.
Berrington, Janet
Bhandarkar, Kailas P
Biondini, Diego
Cappellesso, Rocco
Carlisle, Erica M.
Caulley, Lisa
Chan, Kak-Chen
Chen, Yan
Cura, Jose Luis
de Jesus, Lisieux Eyer
Deshpande, Aniruddh V
Diao, Mei
Djordjevic, Miroslav L
Dorazio, Robert
Esposito, Ciro
Faraoni, David
Federico, Giovanni
Friedmacher, F
Gabra, Hany O S
Gerlach, Undine Ariane
Ghritlaharey, Rajendra Kumar
Gilbert, Alan
Grammatikopoulos, Tassos
Handford, Penny A
Hayes, Roisin

Heise, Carlos O.

Henderson, Lucy

Hirano, Yasuhiro

Hirche, Christoph

Horne, David

Hoshino, Yusuke

Huang, Shoujiang

Iliescu, Dominic

Ishimaru, Tetsuya

Jafar-Nejad, Hamed

Jager, Elzerie de

Jiwane, Ashish

Kamolz, Lars-Peter

Kawahara, Hisayoshi

Kazez, Ahmet

Kessler, Ulf

Khurram, Mohammed F.

Koivusalo, A. I.

Kojima, Atsuhiro

Kumar, Deepak

Kumar, Nitesh

Kuppusamy, Thirumagal

Lal, Richa

Larson, Shawn

Lee, Cho Rok

Leechongs, Somsak

Li, Kai

Li, Long

Lin, Xiao-Kun

Livingston, Michael

Losty, Paul D

Makhija, Deepa

Manji, Karim P.

Marco, Beatriz Bañuelos

Marino, Bruno
McHoney, Merrill

Menahem, Samuel

Méndez-Gallart, Roberto

Meyer, Heidi M.

Meyers, R. L.

Miura, Masaru

Moon, Suk-Bae

Nabi, Zaheer

Nardo, Giovanni Di

Ning, Bo

Olsen, Lars Henning

Osifo, David

Pal, Kamalesh

Paley, Dror

Pars, Hatice

Parsons, Lauren N

Poddighe, Dimitri

Prabu, Ramesh

Pratesi, Claudia B.

Puceat, Michel qazi, saqib

Qvist, Niels

Rao, P. Syamasundar

Rasulic, Lukas

Rathod, Kirtikumar

Rela, Mohamed

Safdari, Farshad

Sahito, Badaruddin

Saleem, Muhammad

Saliba, Aline

Salo, Martin

Salö, Martin

Sarnacki, Sabine

Sarnacki, Sabine

Sato, Takuro

Sharifi-Rad, Lida
Shehata, Sameh

Shin, Hosung

Simmons, M. Abigail

Sinopidis, Xenophon

Soyer, Tutku

Stehr, Maximilian

Surd, Adrian

Sutthatar, Pattamon

Székely, Andrea

Talabi, Ademola Olusegun

Tander, Burak

Tanny, Sharman P Tan

Tartar, Tugay

Thomas, Reju Joseph

Thornton, Paul S.

Tk, Jayakumar

Tou, Jinfa

Valioulis, I.

Varan, Birgul

Vujovic, Katarina Savic

Waisbourd-Zinman, Orith

Wang, Bin

Wang, Yuefu

Weber, Mark D.

Witt, Stefanie

Wong, Kenneth

Woźniak, Magdalena Maria

Yang, Jixin

Yao, Hai-jun yoneda, akihiro

Youn, Joong Kee

Zammit, Stefania Chetcuti

Zani, Augusto

Zavras, Nick

Zhang, Weiping

Zheng, Shan 